\section{"Oumuamua Unpacking the mystery of our interstellar visitor}

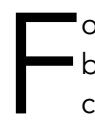

or the time being, the idea of building a spacecraft which can carry us to other stars is a goal reserved for the future - but that doesn't stop interstellar visitors from coming to us. In 2017, a small, mysterious object named 'Oumuamua became the first body in the Solar System ever confirmed to originate from a star system beyond our own.

Immediately, the sighting sparked numerous compelling theories about the origins of 'Oumuamua, and ever since, the story of our understanding of this enigmatic object has followed surprising twists and turns. Although we may never know the full truth, 'Oumuamua could have a lot to tell us about the early history of our Solar System - and how future sightings of interstellar visitors may appear.

\section{AN ODD SPECK IN THE SKY}

Perched on a mountaintop in the Hawaiian island of Maui, the PanSTARRS telescope continually scans the sky for astronomical objects which noticeably move or change in brightness. While searching through data gathered by the telescope, Canadian astronomer Robert Weryk discovered a small object roughly 33 million kilometres from Earth, unlike any

From the beginning, astronomers' observations of the object appeared to make little sense. For a start, its orbit around the Sun had a highly elliptical shape - a type of path typically only followed by comets. However, unlike any normal comet, this body didn't appear to show any sign of a bright, dusty tail.

This left just one explanation: the object must have been ejected from another star system at some point in the distant past, before falling into the Sun's orbit. For this feat, astronomers named Weryk's discovery 'Oumuamua, meaning 'a messenger from the distant past, reaching out to us' in the Hawaiian language. From this point on, the mysteries surrounding 'Oumuamua's origins only deepened.

\section{A SHINY, TUMBLING CIGAR} As they surveyed Pan-STARRS' images, asteroid ever seen before.
Robert Weryk discovered 'Oumuamua using the Pan-STARRS telescope at Haleakalā Observatory, Hawaii, in 2017.

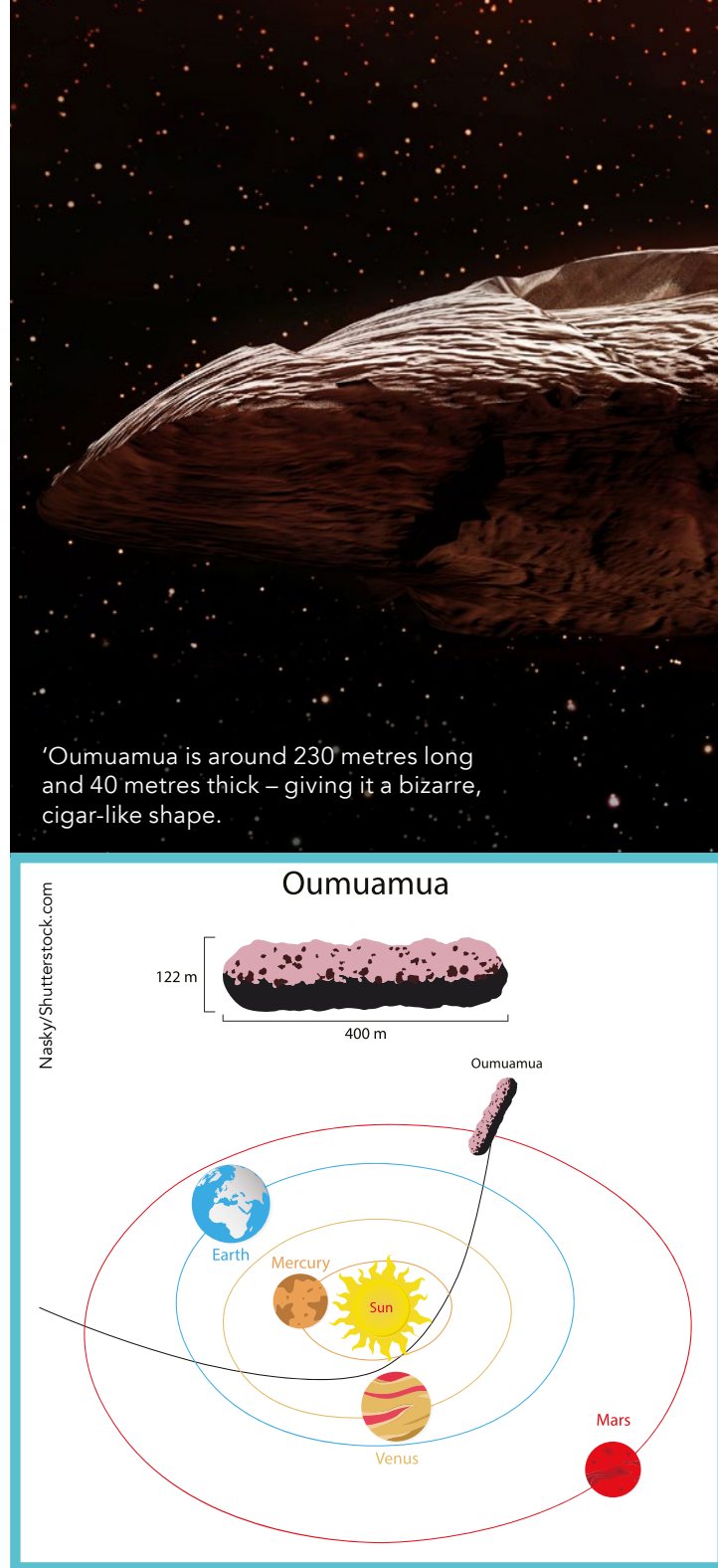

'Oumuamua's orbit around the Sun had a highly elliptical shape - a type of path typically only followed by comets.

researchers noticed that although 'Oumuamua was around 230 metres long, its' thickness was only around 40 metres - giving it a bizarre, cigarlike shape. At the same time, some studies spotted unusual deviations in 'Oumuamua's path, which suggested that it must be propelling itself through space through its own mechanisms.

This was particularly strange since according to some researchers, the forces imparted by this propulsion would cause a body as elongated as 'Oumuamua to spin out of control, and eventually disintegrate. On the contrary, 'Oumuamua appeared to be perfectly stable; rotating in a tumbling motion that didn't threaten to break it apart.

Only adding to the object's strange features was its extreme shininess. As 
